\title{
Hipertensión arterial: estilos de vida y estrategias de intervención
}

\section{Hypertension: lifestyles and intervention strategies}

\author{
Autores/as: \\ Meléndez-Mogollón, Isabel Cristina ${ }^{1}$ \\ García-Silvera, Edgar Edurman ${ }^{2}$ \\ Pérez Arias, Amauris ${ }^{3}$ \\ Vivas Barona, Nicole ${ }^{4}$
}

1. Instituto Tecnológico Superior. Quito, Ecuador. ORCID: https://orcid.org/0000-0002-9030-8935. Email: icmelendez@itslibertad.edu.ec.

2. Instituto Libertad Tecnológico Libertad. Quito, Ecuador. ORCID: https://orcid.org/0000-0001-8116-8427. Email: egarciasilvera@gmail.com.

3. Instituto Libertad Tecnológico Libertad. Quito, Ecuador. ORCID: https://orcid.org/0000-0002-0423-5216. Email: aperez4@istlibertad.edu.ec.

4. Instituto Libertad Tecnológico Libertad. Quito, Ecuador. ORCID: https://orcid.org/0000-0002-4159-7005. Email: nicole.vivas.24@gmail.com.

Puede citar este artículo como: Meléndez-Mogollón IC, García-Silvera EE, Pérez Arias A, Vivas Barona N. Hipertensión arterial: estilos de vida y estrategias de intervención. RECIEN. Revista Científica de Enfermería. 2020; 20: 35-49. https://doi.org/10.14198/recien.2020.20.04

\section{(c) (1)}

Este trabajo se publica bajo una licencia de Creative Commons Reconocimiento 4.0 Internacional (CC BY 4.0) (c) Meléndez-Mogollón IC, García-Silvera EE, Pérez Arias A, Vivas Barona N. 


\section{Resumen}

Introducción: La Hipertensión Arterial (HTA) es una enfermedad crónica de alta prevalencia que debe manejar el paciente en su entorno cotidiano, por lo que el autocuidado se presenta como un elemento de alto valor, y con ello, las prácticas saludables que mantengan las personas.

Objetivo: Evaluar los estilos de vida de personas con HTA, que participan en un programa de intervención comunitaria sobre autocuidado dentro de su entorno rural como parte de una valoración de seguimiento.

Metodología: El diseño es observacional, el nivel descriptivo y de corte transversal. La muestra fue no probabilística de carácter intencional, con la participación de un total de 60 personas que forman parte del "Programa de Autocuidado en Hipertensión Arterial" de la Parroquia de Calderón, Quito, Ecuador. El procesamiento estadístico descriptivo.

Resultados: La media en edad de la población de estudio es de 70.6 años. El 94\% presenta valores de TA e IMC fuera del rango normal. La mayoría de los participantes cumplen sus autocuidados bajo el refuerzo de intervenciones relacionadas a charlas educativas y grupos de apoyo.

Conclusión: Se evidencia la transcendencia del acercamiento instituciones-comunidad y la alfabetización digital, junto a cambios favorables en el estilo de vida, logrando detectar intervenciones significativas para el mantenimiento de los autocuidados.

Palabras clave: Hipertensión arterial; estilos de vida, intervención comunitaria; promoción de salud. 


\section{Abstract}

Introduction: Arterial Hypertension (HBP) is a highly prevalent chronic disease that the patient must manage in their daily environment, so self-care is presented as a high value element, and with it, healthy practices that people maintain.

Objective: To evaluate the lifestyles of people with HT, who participate in a community intervention program on self-care within their rural environment as part of a follow-up assessment.

Methodology: The design is observational, descriptive and cross-sectional. The sample was non-probabilistic of an intentional nature, with the participation of a total of 60 people who are part of the "Self-care Program in Arterial Hypertension" of the Parish of Calderón, Quito, Ecuador. Descriptive statistical processing.

Results: The mean age of the study population is 70.6 years. 94\% present values of BP and $B M I$ outside the normal range. Most of the participants carry out their self-care under the reinforcement of interventions related to educational talks and support groups.

Conclusion: The transcendence of the institutions-community approach and digital literacy is evidenced, together with favorable changes in lifestyle, managing to detect significant interventions for the maintenance of self-care.

Keywords: Arterial hypertension; lifestyle; community intervention; health promotion.

\section{INTRODUCCIÓN}

Las enfermedades cardiovasculares (ECV) son la principal causa de morbilidad y mortalidad a nivel mundial, representan 17.3 millones de muertes a nivel mundial cada año, esta cifra se proyecta en aumento a 23.6 millones por el año 2030(1); en donde el 80 por ciento de estas muertes se producen en zonas bajas y países de ingresos medios ${ }^{(2)}$. 
La incidencia de ECV, incluye enfermedades coronarias, isquemia enfermedad cardíaca, infarto de miocardio, insuficiencia cardíaca congestiva, arrítmias cardíacas como fibrilación auricular, accidente cerebrovascular, periférico enfermedad arterial, aneurisma aórtico, ruptura y disección, tromboembolismo venoso, embolia pulmonar y muerte súbita cardíaca; siendo la Hipertensión Arterial (HTA), un factor de riesgo que incide en todas las enfermedades mencionadas ${ }^{(3)}$.

La tensión arterial indica un aumento de la presión que ejerce la sangre sobre las arterias de la persona y se clasifica en normal (presión arterial sistólica entre 130 y 139 mm Hg o presión arterial diastólica entre 80 y $89 \mathrm{~mm} \mathrm{Hg}$ ), elevada (presión arterial sistólica entre 120 y $129 \mathrm{~mm}$ $\mathrm{Hg}$ o presión arterial diastólica entre 80 y $89 \mathrm{~mm} \mathrm{Hg}$ ) y la hipertensión en estadio 1 y 2 se define como sangre sistólica Presión igual o superior a 130 y $140 \mathrm{~mm} \mathrm{Hg}$, respectivamente o diastólica presión arterial igual o mayor a $90 \mathrm{~mm} \mathrm{Hg}{ }^{(4)}$.

Se estima que en América Latina y el Caribe, el $80 \%$ de las personas con hipertensión no tienen un control adecuado de su presión arterial(5). Según el Estudio Epidemiológico Prospectivo Urbano Rural sobre Hipertensión (PURE) en Argentina, Brasil y Chile, de forma combinada, sólo el $57 \%$ de los hipertensos sabe su condición, mientras que el $53 \%$ se encuentra con tratamiento, a comparación del 30\% que tiene su presión arterial controlada. Sin embargo, el $19 \%$ de personas con su presión controlada son ajenos a el conocimiento de su condición ${ }^{(6)}$ y, de cada 5 personas solo 1 tiene esta situación controlada ${ }^{(5)}$.

En estudios realizados se han determinado que entre hombres y mujeres la prevalencia de la enfermedad es más notoria en hombres que en mujeres hasta la edad de 50 años. Sin embargo, después de esta edad la prevalencia en las mujeres fue superior a los hombres ${ }^{(7-11)}$.

En el Ecuador la tasa de mortalidad es de $6 \%$ por cada 1000 habitantes, de los cuales $7.2 \%$ son hombres y $3.9 \%$ son mujeres, en donde las enfermedades del sistema circulatorio provocaron el $23 \%$ de decesos ${ }^{(12)}$ y de acuerdo al Instituto Nacional de Estadísticas y Censos $^{(13)}$, la HTA representó la cuarta causa de muerte en mujeres y la sexta en hombres de muertes para el 2016. Es alarmante que el 35\% a $40 \%$ de la población adulta con hipertensión arterial, la mitad desconoce que la padece esta enfermedad y menos del $10 \%$ reciben un tratamiento adecuado a su enfermedad(14). 
En el año 2015, la hipertensión arterial y otras patologías superan los 7500 egresos hospitalarios al año, en el 2017 se reportaron 5776 solo por hipertensión primaria, según el registro estadístico de Egresos Hospitalarios del Instituto Nacional de Estadísticas y Censos (INEC) ${ }^{(15)}$.

Ahora bien, la hipertensión es una enfermedad multifactorial, en donde muchos de estos factores son modificables ${ }^{(16)}$. Entre estos factores tenemos la edad, raza, género, Índice de Masa Corporal (IMC), exceso de sal en la dieta, adicción al tabaco, alto consumo de alcohol, deficiencias en la dieta de potasio y calcio, niveles elevados de colesterol y glucosa en sangre, falta de ejercicio, tanto de alto como bajo impacto, y factores psicológicos como el estrés y depresión $^{(17)}$. El objetivo de la investigación se enmarcó en evaluar los estilos de vida de personas con HTA, que participan en un programa de intervención comunitaria sobre autocuidado dentro de su entorno rural como parte de una valoración de seguimiento. En tal efecto, se determinaron los siguientes objetivos específicos: determinar las características sociodemográficas, identificar los antecedentes patológicos, detectar los hábitos de la vida cotidiana y valorar las preferencias de participación comunitaria de las personas involucradas con el proyecto.

\section{METODOLOGÍA}

\section{Diseño y población}

El presente estudio corresponde a un diseño observacional, transversal, con un nivel descriptivo. La muestra fue no probabilística de carácter intencional. Participaron un total de 60 personas (12 hombres y 48 mujeres) en edades comprendidas entre los 45 y 85 años de la Parroquia de Calderón ubicada en el Distrito Metropolitano de Quito (Ecuador), que forman parte del "Programa de Autocuidado en Hipertensión Arterial".

Para dar cumplimiento a la normativa de protección de datos y consentimiento de los pacientes, los responsables del estudio entregaron un consentimiento escrito a cada participante, el cual contenía todos los aspectos detallados de la realización del estudio, su objetivo, descripción, protección de identidad y participación. El consentimiento se consideró como criterio indispensable en la selección de la muestra, lo cual limito el estudio. 
Adicionalmente, los participantes fueron valorados antes de sus actividades dentro del programa de autocuidado, en donde se midió la tensión arterial, el peso y altura permitió calcular el Índice de Masa Corporal (IMC).

\section{Variables e Instrumentos}

Se administró un cuestionario anónimo elaborado por los investigadores de acuerdo a la operacionalización variables de la investigación (Tabla 1).

\begin{tabular}{|c|c|c|}
\hline Variables & Dimensiones & Indicadores \\
\hline \multirow{7}{*}{$\begin{array}{l}\text { Características } \\
\text { sociodemográficas }\end{array}$} & \multirow[b]{2}{*}{ Demográficos } & Edad \\
\hline & & Número personas con que vive \\
\hline & \multirow{5}{*}{ Sociales } & Estado civil \\
\hline & & Área de residencia \\
\hline & & Educación \\
\hline & & Etnia \\
\hline & & Ocupación \\
\hline \multirow{2}{*}{ Estado de Salud } & \multirow{2}{*}{$\begin{array}{c}\text { Valores de } \\
\text { control }\end{array}$} & Tensión Arterial \\
\hline & & Peso y Talla \\
\hline \multirow[b]{2}{*}{ Antecedentes } & $\begin{array}{l}\text { Antecedentes } \\
\text { familiares }\end{array}$ & Registro \\
\hline & $\begin{array}{c}\text { Antecedentes } \\
\text { personales }\end{array}$ & Enfermedades previas \\
\hline \multirow{7}{*}{$\begin{array}{l}\text { Hábitos de la vida } \\
\text { cotidiana }\end{array}$} & Actividad física & Indicaciones médicas \\
\hline & $\begin{array}{l}\text { Descanso y } \\
\text { sueño }\end{array}$ & Frecuencia \\
\hline & $\begin{array}{l}\text { Signos de } \\
\text { alerta }\end{array}$ & Autovaloración \\
\hline & $\begin{array}{c}\text { Cumplimiento } \\
\text { de } \\
\text { indicaciones } \\
\text { médicas }\end{array}$ & Frecuencia \\
\hline & $\begin{array}{l}\text { Prácticas } \\
\text { recreativas }\end{array}$ & Frecuencia \\
\hline & Alimentación & Control \\
\hline & Higiene & Frecuencia \\
\hline \multirow{3}{*}{$\begin{array}{l}\text { Preferencias de } \\
\text { participación }\end{array}$} & $\begin{array}{l}\text { Actividades } \\
\text { recreativas } \\
\text { sociales }\end{array}$ & Frecuencia \\
\hline & $\begin{array}{l}\text { Espacios de } \\
\text { participación } \\
\text { comunitaria }\end{array}$ & frecuencia \\
\hline & $\begin{array}{c}\text { Uso de } \\
\text { medios } \\
\text { tecnológicos }\end{array}$ & frecuencia \\
\hline
\end{tabular}

Tabla 1. Operacionalización de las variables de la investigación. 
La validación se efectuó a través de la valoración de tres expertos, de acuerdo a la normativa para validación de instrumentos por juicio de expertos establecida por el Comité de Investigación del Instituto Superior Tecnológico Libertad. El cuestionario fue entregado en su sobre sellado a cada participante con la presencia del líder comunitario.

\section{Tratamiento de los datos}

Para la codificación y el respectivo análisis estadístico de los datos se utilizó el programa informático IBM SPSS v.25.0. Statistics (IBM Corporation, USA) para Windows. La estadística descriptiva se mostró como media y desviación estándar para las variables continuas y en términos de frecuencia y porcentajes para las variables categóricas.

\section{RESULTADOS}

Durante el período de estudio se examinaron 60 personas de 45 a 85 años. Los datos demográficos de los participantes se resumen en la Tabla 2.

\begin{tabular}{|c|c|c|c|c|}
\hline & Media & Moda & Curtosis & $\begin{array}{l}\text { Coeficiente de } \\
\text { asimetría }\end{array}$ \\
\hline Población & $70.61 \pm 1.1$ & 77 & -0.34 & -0.55 \\
\hline $\begin{array}{c}\text { Números de } \\
\text { personas con que } \\
\text { vive }\end{array}$ & $3 \pm 0.4$ & 2 & 3.78 & 1.73 \\
\hline \multirow[t]{3}{*}{ Estado civil (\%) } & casado & viuda & divorciado & otros \\
\hline & 61.7 & 13.3 & 8.3 & 16.7 \\
\hline & Sin estudios & $\begin{array}{l}\text { Básica } \\
\text { completa }\end{array}$ & $\begin{array}{l}\text { Bachiller } \\
\text { completo }\end{array}$ & Superior completa \\
\hline Educación (\%) & 66.6 & 21.7 & 5.0 & 6.7 \\
\hline
\end{tabular}

La media en edad de la población de estudio es de 70.6 años, siendo la edad con más reiteración en el estudio de 77 años. 
En el caso del número de personas con que vive, la media fue de 3 personas, siendo dos la que más se reiteraba en los hogares. Los valores de curtosis y coeficiente de asimetría positivo indican que los datos presentan curtosis leptocrítica, no hay mucha variación en los resultados y el sesgo de los datos se distribuyen a la izquierda de la media. El mayor porcentaje de los encuestados están casados (61.7\%), mientras que los estudios completados se distribuyen en superior, bachiller y básica completa. La mayoría de los encuestados (66.6\%) no presenta estudios.

En la figura 1, se representa un análisis del Índice de Masa Corporal (IMC) y la Tensión Arterial (TA).

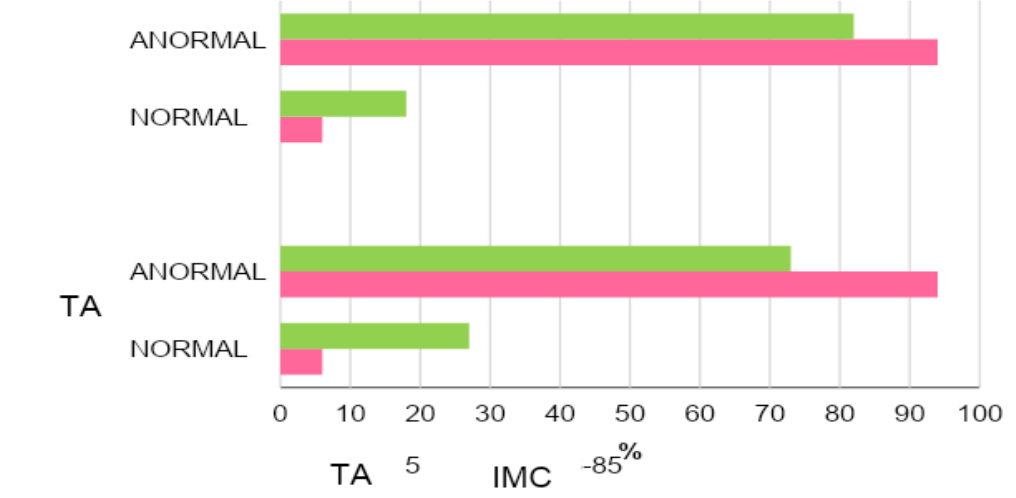

Figura 1. Relación entre el Índice de Masa Corporal (IMC) y la Tensión Arterial (TA). Índice de Masa Corporal normal: 18 a 25.

Tensión Arterial normal: 120/80 mmHg.

Los datos referentes al IMC y la TA, el $6 \%$ de los encuestados de 45 a 65 años se encuentra dentro del rango adecuado mientras que el $94 \%$ restante de encuestados se encuentra fuera del rango aceptado. Respecto a los encuestados de 66 a 85 años se presenta un $27 \%$ normal y un $73 \%$ fuera del rango.

En referencia a la TA, el $6 \%$ de encuestados entre los 45 a 65 años se encuentra normal, mientras que el $94 \%$ se encuentra fuera de los valores normales, el 18\% entre los 66 a 85 años están dentro de los valores normales, por ende, el $82 \%$ está fuera del rango considerado normal. Para analizar el estilo de vida por género de los pacientes se analizó la frecuencia de actividades físicas, con alto impacto (con esfuerzo) y bajo impacto (mínimo esfuerzo) (Fig. 2). 


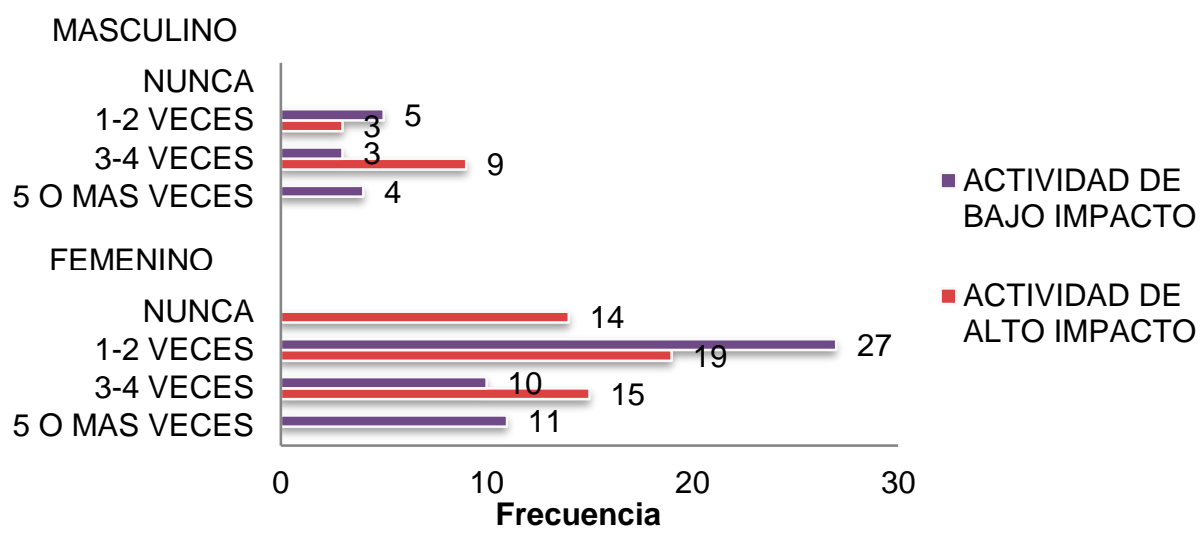

Rango de edad en estudio 45-85

Figura 2. Participación en actividades de alto y bajo impacto.

En la figura 2 se observa que tanto hombres como mujeres, realizan actividades de alto y bajo impacto, en donde, mayoritariamente se encuentran las mujeres ya que son las predominantes en el grupo de estudio a comparación de los hombres. Se observa que las mujeres prefieren realizar actividades de bajo y alto impacto con una frecuencia de 1 a 2 veces por semana, contrario a los hombres, los cuales optan por realizar actividades de alto impacto 3 o 4 veces por semana y actividades de bajo impacto 1 a 2 veces por semana.

En cuando a las actividades para favorecer la salud de los encuestados, la selección según preferencia fue el baile (24\%) seguido de oír música (23\%), mientras un $16 \%$ indico las manualidades, un $14 \%$ el canto y las terapias de relajación y solo un $9 \%$ prefiere pintar.

\begin{tabular}{|c|c|c|c|c|c|c|c|c|c|}
\hline $\begin{array}{l}\text { Estrato de } \\
\text { edad por } \\
\text { género }\end{array}$ & Escala & $\begin{array}{l}\text { Asiste a } \\
\text { reuniones }\end{array}$ & $\begin{array}{l}\text { Asiste a la } \\
\text { iglesia }\end{array}$ & $\begin{array}{c}\text { Mira } \\
\text { televisión }\end{array}$ & Lee & $\begin{array}{c}\text { Usa la } \\
\text { computadora }\end{array}$ & $\begin{array}{l}\text { Practica } \\
\text { algún } \\
\text { deporte }\end{array}$ & Baila & $\begin{array}{l}\text { Yoga o } \\
\text { taichí }\end{array}$ \\
\hline \multirow{3}{*}{$\begin{array}{c}\text { Femenino } \\
45-65\end{array}$} & $F$ & 12,8 & 28,2 & 15,8 & 20,5 & 2,6 & 5,2 & 15,8 & 0 \\
\hline & AV & 18,8 & 6.6 & 24,4 & 9,9 & 9,9 & 15,5 & 18,8 & 0 \\
\hline & $\mathrm{N}$ & 8,3 & 4,6 & 2,8 & 8,3 & 22,9 & 16,6 & 6,5 & 31,5 \\
\hline \multirow{3}{*}{$\begin{array}{c}\text { Femenino } \\
66-85\end{array}$} & $\mathrm{~F}$ & 17,6 & 28,7 & 25,7 & 13,5 & 1,35 & 5,4 & 8,10 & 0 \\
\hline & AV & 18,9 & 13,9 & 13,9 & 13,9 & 1,4 & 10,4 & 27,8 & 1,4 \\
\hline & $\mathrm{N}$ & 3,8 & 0 & 1,9 & 10,67 & 30,9 & 19,4 & 2,9 & 31,6 \\
\hline \multirow{3}{*}{$\begin{array}{l}\text { Masculino } \\
45-65\end{array}$} & $F$ & 0 & 33,3 & 33,3 & 0 & 0 & 33,3 & 0 & 0 \\
\hline & AV & 16,6 & 0 & 16,6 & 16,6 & 0 & 16,6 & 33,3 & 0 \\
\hline & $\mathrm{N}$ & 14,8 & 14,8 & 0 & 14,28 & 28,7 & 0 & 0 & 28,7 \\
\hline \multirow{3}{*}{$\begin{array}{c}\text { Masculino } \\
66-85\end{array}$} & $\mathrm{~F}$ & 12 & 24 & 20 & 24 & 0 & 8 & 12 & 0 \\
\hline & AV & 24 & 16 & 16 & 12 & 0 & 8 & 24 & 0 \\
\hline & $\mathrm{N}$ & 3,3 & 0 & 3,3 & 3,3 & 33,3 & 20 & 3,3 & 33,3 \\
\hline
\end{tabular}

Tabla 3. Actividades recreativas sociales preferidas por la población de estudio. Abreviaturas F: Frecuentemente/AV: Algunas veces/N: Nunca.

Los datos en la tabla están referidos en porcentaje. 
Según los datos obtenidos en la tabla 3 se observa que las mujeres encuestadas de 45 a 65 años prefieren asistir a la iglesia de forma frecuente representando un $28,2 \%$, mientras que mirar televisión y bailar serían las segundas alternativas más seleccionadas representando un $15,8 \%$, las mujeres de 66 a 85 años de igual manera optan por ir a la iglesia de manera frecuente representando $28,7 \%$ y solo el $13,9 \%$ asiste algunas veces, las otras dos opciones más seleccionadas fue el mirar televisión con un $25,7 \%$ y un $17,6 \%$ eligieron asistir a reuniones con amigos.

Los hombres de 45 a 65 años optaron por 3 opciones más frecuente, estas son ir a la iglesia, mirar televisión y practicar deporte, en donde representa cada una a un 33,3\%, mientras que los hombres de 66 a 85 años prefieren leer y asistir a la iglesia de manera frecuente representando cada una un $24 \%$. Tanto en mujeres como en hombres la mayoría de la población prefiere asistir a la iglesia en comparación con las otras actividades. La mayoría de la población opta por socializar, siendo superior en un $60 \%$, los que prefieren actividades colectivas (asistencia a reuniones, iglesia, deportes, bailes) y fue muy equitativa entre mujeres y hombres y por estrato de edad.

Las personas encuestadas prefieren la participación comunitaria a través de charlas educativas (39\%), asistir a una consulta médica $(22 \%)$ y pertenecer a un grupo de apoyo representando el $(21 \%)$, respectivamente del total de la población. El de menor acogida fue el de recibir asesoría virtual con un $4 \%$ de aceptación. Es interesante que el $60 \%$ de los encuestados prefieren practicas compartidas, grupales (charlas educativas, pertenecer a grupo de apoyo).

En cuanto al uso de medios tecnológicos, los resultados indican que el 34\% no seleccionó ninguna opción, los medios tradicionales como el televisor y radio fue del $21 \%$ y $11 \%$ respectivamente. Medios más avanzados como el celular y el internet, fue del 28 y $6 \%$. 


\section{DISCUSIÓN}

La población de estudio tuvo un promedio de 70 años, la esperanza de vida en Ecuador es de 76 años, estando en el rango medio alto en el mundo, razón por la que se considera una población envejecida ${ }^{(18)}$. Este aspecto es de particular importancia si se tiene en cuenta que los resultados obtenidos en el presente trabajo la mayoría de la población presenta sobrepeso y obesidad (94\%). La prevalencia de obesidad y sobrepeso ha aumentado rápidamente en todas las regiones del mundo, y esto es uno de los factores de riesgo en la HTA(19).

Los valores de curtosis y coeficiente de asimetría negativa indican que hay una gran variabilidad en los datos y que el sesgo de los datos se encuentra más desplazados hacia la media en adelante, lo que represento una limitación dentro del estudio debido a que la muestra estaba sujeta a la voluntad de las personas a participar en el programa y estudio, y la homogeneidad en cuanto a la características, no se pudo controlar, lo que se relaciona íntimamente a la tendencia de afectación global en esta patología(2).

Los cambios en el estilo de vida tienden a no ocurrir de forma aislada, pues en personas con HTA, se requiere modificar las actividades socio-recreativas, inactividad física, tabaquismo, dieta poco saludable ${ }^{(20)}$. La adopción de una vida saludable facilita la pérdida de peso, aumenta la capacidad de respuesta a los medicamentos antihipertensivos.

Las actividades que más le gustaría realizar a los participantes fue bailar y oír música, el aplicarlas beneficiará la salud de los encuestados permitiéndoles tener un mejor estilo de vida y mejorar de igual manera su estado emocional, no obstante, existen otros factores como la dieta que depende del nivel económico de las personas ${ }^{(20)}$.

Existe una relación directa entre el vínculo del individuo y la comunidad, factores de organización social con las enfermedades crónicas como la HTA ${ }^{(21)}$. En el presente estudio se describe que los pacientes optan por tener un nivel de socialización alto, más del $60 \%$ de actividades sociales antes de realizar actividades individuales en todos los estratos de edad. La distribución de estas actividades tendió a ser equitativa, por lo que le gustan realizar diversas actividades de manera cotidiana. La promoción de la salud representa una política social y política integral abarcando acciones dirigidas a fortalecer las habilidades y capacidades de los individuos en el tratamiento de enfermedades, sino también conlleva a cambiar las condiciones sociales, ambientales y económicas del paciente ${ }^{(17)}$. 
Los resultados obtenidos describen que la aplicación de la intervención la prefieren compartida, en lugar de individualmente. Las charlas educativas actuarán de mejor manera para algún tipo de capacitación. Esto asocia la necesidad de interactuar entre sí con otras personas, de socializar, aunque también es favorable desarrollar habilidades individualmente ${ }^{(21)}$.

En la actualidad se vive en aislamiento y distanciamiento social debido al COVID-19, lo cual revaloriza la intervención por medio de las redes sociales, pues el uso generalizado y la influencia de Internet y las redes sociales a nivel mundial representa nuevas oportunidades de vínculos y desafíos para la promoción de la salud. No obstante, en la exploración de este aspecto, la mayoría de la población no optaría por ninguna alternativa ya que no las consideran tan fiables, pero existe un resultado equitativo en usar los medios tradicionales como TV y radio respecto al uso del celular e internet (33\%), lo que lleva a considerar el explorar otras variables que no fueron medidas en el estudio.

\section{CONCLUSIONES}

En la investigación realizada se encontró que las personas a pesar de ser hipertensos bajo un control presentan un alto IMC. Dentro de las actividades que prefieren realizar son de tipo social, donde exista interrelación con otras personas. Una propuesta de, (ya que es en relación a los datos obtenidos no evaluando si realmente esa propuesta es la mejor o no) metodología para intervenir en el estilo de vida de las personas hipertensas de Calderón, es hacerlo mediante charlas educativas y que intervengan actividades como el baile la música. Es necesario para futuras investigaciones e intervenciones trabajar en el acercamiento y alfabetización digital para realizar las intervenciones. 


\section{REFERENCIAS BIBLIOGRÁFICAS}

1. Organización Mundial de la Salud. Nota descriptiva: Enfermedades cardiovasculares. Datos y cifras. [Internet]. 17 de mayo de 2017 [cited 2019 diciembre 20. Recuperado de: https://www.who.int/es/news-room/fact-sheets/detail/cardiovascular-diseases-(cvds)

2. Organización Mundial de la Salud. Informe sobre la situación mundial de las enfermedades no transmisibles 2010. [Internet]. 2011 [cited 2020 enero 12. Recuperado de: https://www.who.int/nmh/publications/ncd_report_summary_es.pdf?ua=1

3. Roth, et al. Global, Regional, and National Burden of Cardiovascular Diseases for 10 Causes, 1990 to 2015. Journal of the American College of Cardiology. [Internet]. 2017. Recuperado de: https://doi.org/10.1016/j.jacc.2017.04.052

4. Kearney PM, Whelton M, Reynolds K, Muntner P, Whelton PK, He J. Global burden of hypertension: analysis of worldwide data. Lancet [Internet]. 2005 [Jan 15-21]; 365(9455):21723. PMID: 15652604. Recuperado de: https://doi.org/10.1016/S0140-6736(05)17741-1

5. Organización Mundial de la Salud. Notas descriptivas: Hipertensión. [Internet].; 13 de septiembre de 2019 [cited 2020 enero 12]. Recuperado de: https://www.who.int/es/news$\mathrm{room} / \mathrm{fact}-\mathrm{sheets/detail/hypertension}$

6. Organización Panamericana de la Salud. La OPS/OMS pide dar más atención al control de la hipertensión. [Internet]. 2015. Recuperado de: https://www.paho.org/hq/index.php?option=com_content\&view=article\&id=10957:2015-pahourges-more-attention-to-blood-pressure-control\&ltemid=1926\&lang=es

7. Tagle V Rodrigo, Acevedo Mónica, Valdés Gloria. Hipertensión arterial en la mujer adulta. Rev. méd. Chile [Internet]. 2013 Feb [citado 2021 Mar 27]; 141(2):237-247. Recuperado de: https://doi.org/10.4067/S0034-98872013000200014

8. Gupta AK, Nasothimiou EG, Chang CL, Sever PS, Dahlöf B, Poulter NR. Baseline predictors of resistant hypertension in the Anglo-Scandinavian Cardiac Outcome Trial (ASCOT): a risk score to identify those at high-risk. [Internet]. 2011. Recuperado de: https://doi.org/10.1097/HJH.0b013e32834a8a42 
9. Solini A, Zoppini G, Orsi E, Fondelli C, Trevisan R, Vedovato M, et al. Resistant hypertension in patients with type 2 diabetes: clinical correlates and association with complications. 32(12):2401-10; discussion 2410. [Internet]. 2014 Dec. Recuperado de: https://doi.org/10.1097/HJH.0000000000000350

10. EUGenMed, Cardiovascular Clinical Study Group, Regitz-Zagrosek V, Oertelt-Prigione S, Prescott E, Franconi F, Gerdts E, et al. Gender in cardiovascular diseases: impact on clinical manifestations, management, and outcomes. Eur Heart J. 2016 Jan 1; 37(1):24-34. Epub [Internet]. 2015 Nov 3. PMID: 26530104. Recuperado de: https://doi.org/10.1093/eurheartj/ehv598

11. Hanus KM, Prejbisz A, Gąsowski J, Klocek M, Topór-Mądry R, Leśniak W, et al. Relationship between gender and clinical characteristics, associated factors, and hypertension treatment in patients with resistant hypertension. Kardiol Pol. 75(5):421-431. [Internet]. 2017. Recuperado de: https://doi.org/10.5603/KP.a2017.0020

12. World Healt Organization. Healt in the Americas. Country Report: Ecuador. [Internet]. 2017. Recuperado de: https://www.paho.org/salud-en-las-americas2017/?page_t_es=informes-de-pais/ecuador

13. Instituto Nacional de Estadísticas y Censos. Estadísticas Vitales. Registro Estadístico de Nacidos Vivos y Defunciones. [Internet]. 2016. Recuperado de: https://www.ecuadorencifras.gob.ec/documentos/webinec/Poblacion_y_Demografia/Nacimientos_Defunciones/2016/Presentacion_Nacimientos_y Defunciones_2016.pdf

14. Freire et al. Tomo I: Encuesta Nacional de Salud y Nutrición de la población ecuatoriana de cero a 59 años. ENSANUT-ECU 2012. Ministerio de Salud Pública/Instituto Nacional de Estadísticas y Censos. Quito-Ecuador. [Internet]. 2014. Recuperado de: https://www.ecuadorencifras.gob.ec/documentos/webinec/Estadisticas_Sociales/ENSANUT/MSP_ENSANUT-ECU_06-10-2014.pdf

15. Coello C. Ecuador tiene una prevalencia de 9,3 por ciento de hipertensión. Edición Médica. Profesionales. [Internet]. 2019.2 Recuperado de: https://www.edicionmedica.ec/secciones/profesionales/ecuador-tiene-una-prevalencia-de-93-por-ciento-de-hipertension-94139 
16. Campos-Nonato I, Hernández-Barrera L, Pedroza-Tobías A, Medina C, Barquera S. Hipertensión arterial en adultos mexicanos: prevalencia, diagnóstico y tipo de tratamiento. Ensanut MC 2016. Salud Publica Mex; 60:233-243. [Internet]. 2018. Recuperado de: https://doi.org/10.21149/8813

17. Alston L, Peterson KL, Jacobs JP, Allender S, Nichols M. Quantifying the role of modifiable risk factors in the differences in cardiovascular disease mortality rates between metropolitan and rural populations in Australia: a macrosimulation modelling study. BMJ Open; 7(11): e018307. [Internet]. 2017. Recuperado de: https://doi.org/10.1136/bmjopen-2017018307

18. Datos Macro. Expansión sociodemografica. [Internet]. 2019. Recuperado de: https://datosmacro.expansion.com/demografia/

19. Tanaka M, Itoh H. Hypertension as a Metabolic Disorder and the Novel Role of the Gut. Curr Hypertens Rep.; 21(8): 63. [Internet]. 2019. Recuperado de: https://doi.org/10.1007/s11906-019-0964-5

20. Mozaffarian D, Hao T, Rimm EB, Willett WC, Hu FB. Changes in diet and lifestyle and long-term weight gain in women and men. N Engl J Med. 364(25):2392-404. [Internet]. 2011. Recuperado de: https://doi.org/10.1056/NEJMoa1014296

21. Thorolfur T. Bring in the social context: Towards an integrated approach to health promotion and prevention. Scandinavian Journal of Public Health, 39(Suppl 6): 19-25. [Internet]. 2011. Recuperado de: https://doi.org/10.1177/1403494810394549 